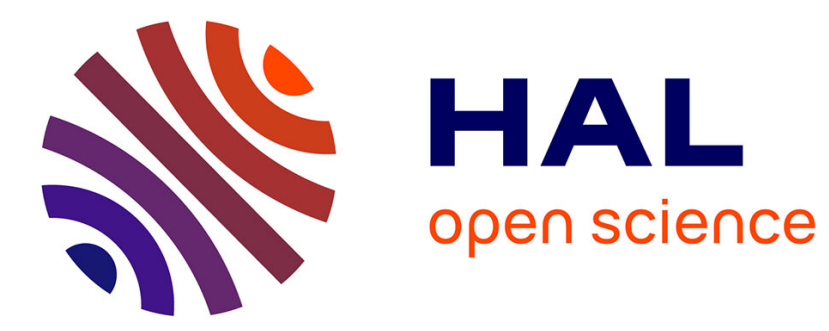

\title{
Adaptive flow control laws: A simulation based comparison with low order models
}

Cédric Raibaudo, Philippe Mouyon, Carsten Doll

\section{To cite this version:}

Cédric Raibaudo, Philippe Mouyon, Carsten Doll. Adaptive flow control laws: A simulation based comparison with low order models. AIAA Aviation 2019 Forum, Jun 2019, Dallas, United States. 10.2514/6.2019-2913 . hal-02992565

\section{HAL Id: hal-02992565 \\ https://hal.science/hal-02992565}

Submitted on 6 Nov 2020

HAL is a multi-disciplinary open access archive for the deposit and dissemination of scientific research documents, whether they are published or not. The documents may come from teaching and research institutions in France or abroad, or from public or private research centers.
L'archive ouverte pluridisciplinaire HAL, est destinée au dépôt et à la diffusion de documents scientifiques de niveau recherche, publiés ou non, émanant des établissements d'enseignement et de recherche français ou étrangers, des laboratoires publics ou privés. 


\title{
Adaptive flow control laws: A simulation based comparison with low order models
}

\author{
C. Raibaudo* \\ Ph. Mouyon ${ }^{\dagger}$ \\ C. Döll $l^{\ddagger}$ \\ ONERA - DTIS, Toulouse, 31055, France
}

\section{Introduction}

In the past few decades, the control of turbulent boundary layers separation has been crucial for aerodynamic performances improvement ([1]). Passive devices, like vortex generators, have been first considered for an efficient redistribution of the energy between the external fluid and the separation region ([2]). The separation process is yet sensitive to unsteady upstream perturbations and related to the absolute instability of the flow ([3]). Active flow control (AFC) is therefore preferred and offers more flexibility in the control parameters. An adaptation of the airfoil shape to the speed or the weight could also increase the performances during the flight. For this objective, a multi-disciplinary research platform involving European partners has been created for the development of an electro-active morphing wing. Both low-frequency camber control and high frequency vibrating trailing edge (HFVTE) serve as actuators for the wing. The HFVTE in particular will be used for the feedback control. The actuator vibration is about the millimeter in amplitude and up to $200 \mathrm{~Hz}$ in frequency. Pressure taps are also located upstream to the leading edge on the upper surface. Two $6 \mathrm{~mm}$ diameter microphones and one dynamic pressure sensor (Kulite XCQ063) are put to measure the characteristic state of the flow under control in real-time. More details on the actuators and preliminary open-loop results can be found in [4] and [5].

In order to increase the robustness and maintain performances despite disturbances and uncertainties, active flow control should be performed in closed-loop control. The design of a closed control law is generally based on a system model of rather low order. This model must keep the main characteristics of the flow, with its control input and output. High non-linear behavior of flows, disturbances and uncertainties on the model (on speed, pressure, temperature, Reynolds number...) make model-based control more difficult to achieve ([6]). Adaptive methods are therefore useful techniques to deal with these unmodeled effects. Extremum-seeking $([6,7])$ and slope-seeking $([8,9])$ have been used in the past for flow control applications, but more progress could be achieved in this field. Different techniques can be considered: (i) a model free approach using polynomial approximation of the dynamics ([10]), (ii) direct adaptive control consisting on tuning the gains on-line of a feedback loop in order to minimize a given criterion ([11]) and (iii) indirect adaptive control consisting on estimate on-line a system model and use it to design the control law ([12]). The present study will focus on the analysis of the natural flow dynamics of the experimental morphing wing in order to obtain low order dynamical models, as attractors, and the development of direct adaptive techniques for the stabilization of the flow in closed-loop control.

Figure 1 shows the main ingredients of the simulation developed. Two reduced order models of the flow are suggested. The first one is the classical nonlinear Van der Pol oscillator. The second one is based on the coupling of the previous one with a filtered chaotic oscillator (the May-Feigenbaum oscillator). This yields to a more realistic representation of the measurement signal, with a broadband spectrum.

Using these models, several controllers are developed and compared their performances. We first highlight the limitation of the simple control laws conventionally used to suppress the oscillations of Van der Pol models. An advantage of these laws is that it is relatively easy to adapt them in real time. We describe and test many of these adaptation procedures. Then we introduce more sophisticated control laws (dynamic and not static

*Post-Doctorant, ONERA-DTIS, 2, avenue Edouard Belin, 31055 Toulouse Cedex 4, France.

$\dagger$ Expert, ONERA-DTIS, 2, avenue Edouard Belin, 31055 Toulouse Cedex 4, France.

${ }^{\ddagger}$ Senior Specialist, ONERA-DTIS, 2, avenue Edouard Belin, 31055 Toulouse Cedex 4, France. 


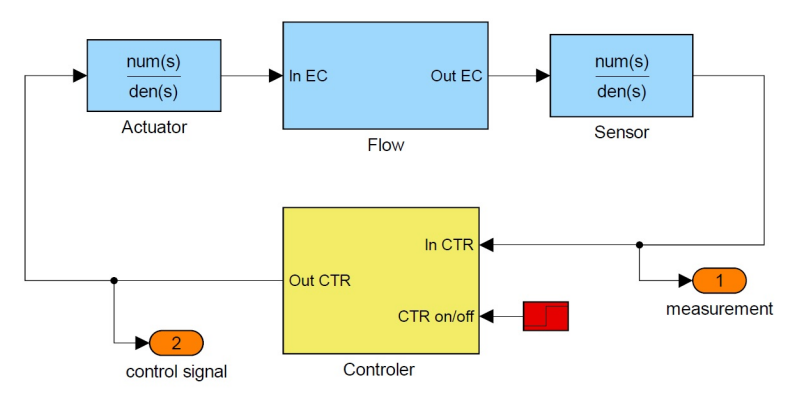

Figure 1: Block diagram for the flow control.

controllers). The adaptation of these command laws is however more touchy. Some ways to implement the controller are to be favoured to facilitate its adaptation and avoid the occurrence of instability flares. This is already known in adaptive filtering of signals, but amplified here by the fact of working in closed loop. Finally, an architecture with a double adaptation is proposed. It makes it possible to regulate the power of the injected control signal on the one hand, and on the other hand to adjust the dynamic behavior.

\section{Approximated model of the flow for the law design}

Fluids usually consist of multiple-frequency dynamics. For the configuration considered, significant frequencies have been found in the PSD of temporal coefficients modes of the POD corresponding to different physical phenomena in the airfoil wake: the Von Kármán vortex shedding at a dimensionless frequency $F^{*}=f c / U_{\infty}=8.5$, a higher order mode dynamics at $F^{*}=14.1$, a low-frequency shift mode linked to the shear-layer at $F^{*}=3.7$, etc. ([5]). In order to design closed loop control laws we use a model that approximated the behavior of the flow along with its actuators and sensors. The model must not be too much complex so that it can be used easily for control design purpose. Nevertheless it must capture the main characteristics of the phenomenon to be control. In the present case the control is intended to reduce intrinsic vibratory phenomenons that are present in the flow model. A careful consideration of the flow frequencies in this low-order model used for the simulation is then crucial. Nonlinear oscillators such as the Van der Pol (VdP) oscillator are therefore an satisfying choice. Van der Pol non-linear oscillator is chosen from previous works as able to represent flow stability and dynamics ([13]). This model has been used to reproduce and control the dynamics of simplified bluff bodies ([14]) or airfoils ([15]) for example.

The difficulty we encountered in order to work in 3D complex flows comes in part from the fact that the spectrum of the measures in 3D is broadband and no longer narrow band as in 2D. In order to model such a behavior we introduce a nonlinear chaotic oscillator that is known to deliver a broadband signal still in an intrinsic fashion. Coupling the two oscillators yields to a more realistic model of the flow to be control. Pressure dynamics on the experimental airfoil will be acquired to design low-order VdP models with corresponding frequencies of oscillation.

\section{A. Quasi-harmonic nonlinear oscillator}

The state space representation for the controlled Van der Pol model is expressed as:

$$
\left\{\begin{array}{l}
\dot{x}_{1}=x_{2}+G u \\
\dot{x}_{2}=-\omega_{0}^{2} x_{1}+\left(2 \xi \omega_{0}-3 K_{s} x_{1}^{2}\right)\left(x_{2}+G u\right) \\
y=x_{1}
\end{array}\right.
$$

where $x_{1}, x_{2}$ are the states of the system, $y$ is the measured output, and $u$ is the control input.

System (1) can be rewritten as a second order ordinary differential equation:

$$
\ddot{y}-2 \xi \omega_{0} \dot{y}+3 K_{s} y^{2} \dot{y}=G \omega_{1} \cos \omega_{1} t .
$$

The determination of the constants is realized using the permanent regime response. More details can be found in [13]. 

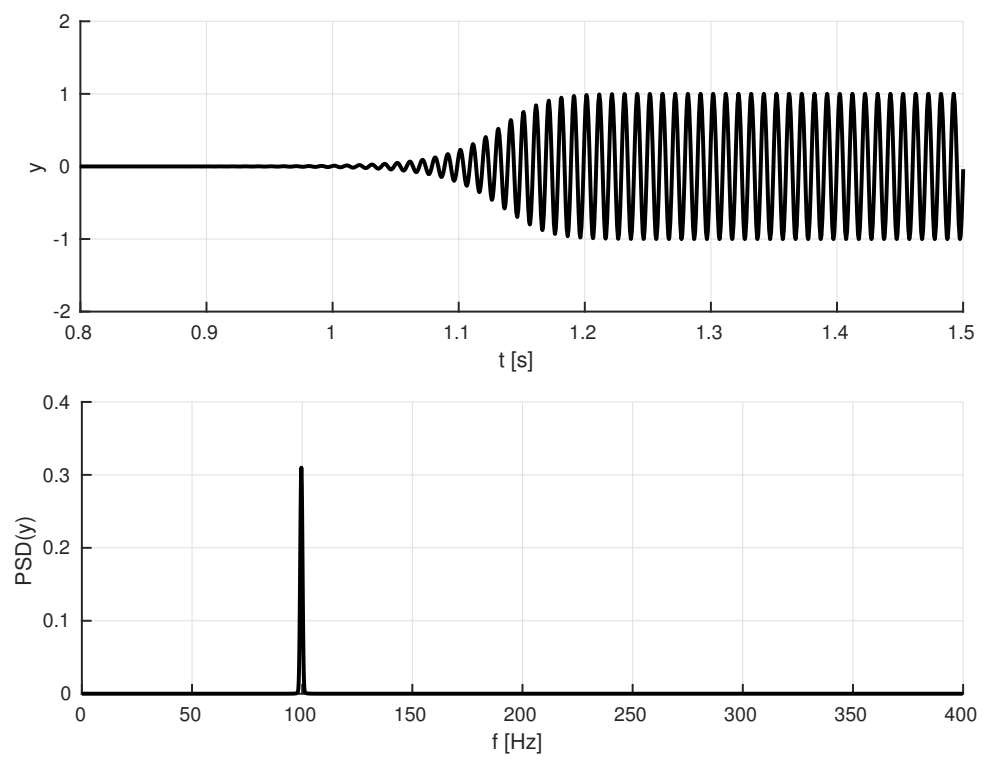

Figure 2: Time history and PSD of the unforced response with self-sustained oscillations (from [13]).

Figure 2 shows the time history and PSD of the response of the unforced VdP model. A sinusoidal input of amplitude 1 and frequency $50 \mathrm{~Hz}$ is considered here. The interest of the VdP oscillator is the self-sustained oscillations are maintained even when the actuation is off.

\section{B. Coupled Van der Pol and May-Feigenbaum oscillators}

The coupled oscillators model is depicted on the figure 3. The May-Feigenbaum block is a chaotic oscillator tuned so that it deliver a stochastic signal with an almost flat spectrum over all the frequency band. The low-pass filter is intend to limit this frequency band. The product with the oscillating output of the quasiharmonic Van der Pol model will results in a shift of the May-Feigenbaum output spectrum around the intrinsic frequency of the Van der Pol model (see figure 4).

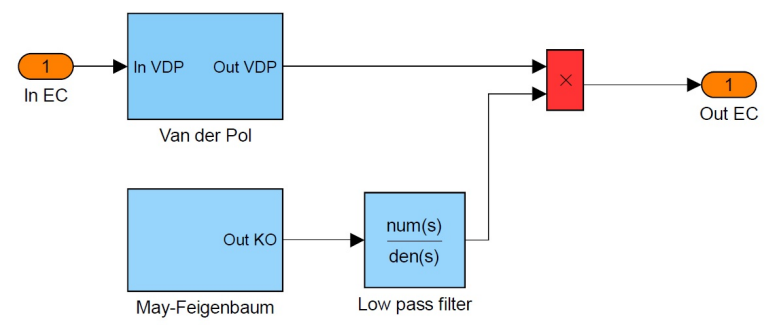

Figure 3: Block diagram for the flow model

\section{Adaptive control law structures}

Several adaptations procedure have been developed. They are all based on an architecture composed of four blocks (see figure 5).

- A first block is used on practical expriments in order to formate the aerodynamic measurements delivered by the sensors into the signal $y$. It may consist in a simple scaling, but could be far more complex depending on the physics of the sensing process. 


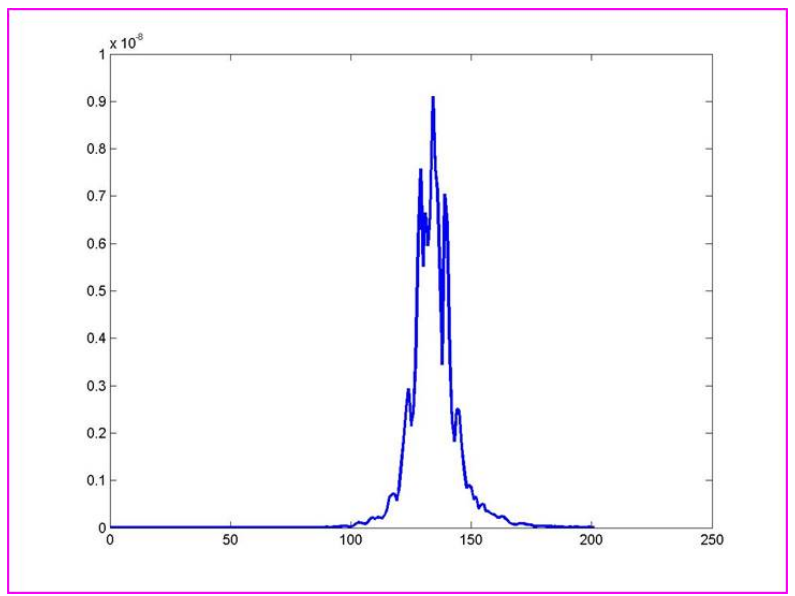

Figure 4: Coupled oscillators: spectrum of the output

- A second block evaluates a performance criterion $J$ calculated from the measurements (We often use a nonlinear filter that estimates the instantaneous standar deviation of the sensed signals).

- A thrid block optimizes the parameters of the control law in order to minimize this criterion.

- And a last bloc is the computation of the control signal $u$ itself that will be fed to the actuator.

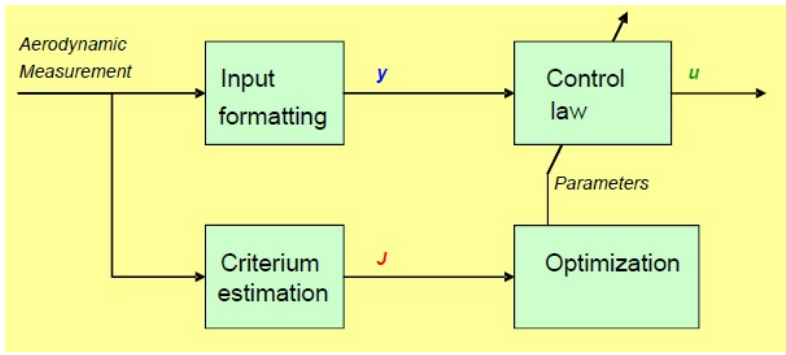

Figure 5: The overall control architecture

\section{A. Static gain controller}

Static output feedback control $u=K y$ is known to be able to suppress oscillations of Van der Pol models. Indeed, a Van der Pol oscillator is a linear unstable dynamical system, along with a non linear feedback that that prevents the divergence when the amplitude of the oscillations becomes too great. Thus any linear feedback stabilizing the linear dynamic will remove the limit cycle.

If the characteristics of the Van der Pol model are not well known, and especially if its input gain $g$ is unknown it is not possible to found the value of the feedback gain $K$ that suppresses the oscillations. An online adaptation of $K$ may used for that purpose. Furthermore if the Van der Pol gain $g$ varies the adaptation will adjust $K$ in order to still remove the oscillations.

Because we work on a very simple simulation model, there is no need of any measurement formatting block. The output of the simulated flow is $y$. The criterium evaluation will be detailled in the paper. The calculations made in the control law block are reduced to a simple multiplication $u=K y$.

Several adaptations procedures have been developed optimizes the parameters of the control law (here the gain $K$ ) in order to minimize this criterion. The paper will describes some of them:

- Gradient descent. The main difficulty is to find en efficient way to compute the criterium gradient.

- Local grid optimization. The optimization process reduces to the choice of the tuning minimizing the criterium. To acheive good performances, the grid must adapted online. 


\section{B. Delay controller}

Another well known way used to removes Van der Pol oscillatory behavior is to implement a static feedback applied to the delayed measurement, i.e. $u(t)=K y(t-T)$. In such a case the gain is kept constant but the delay $T$ is to be tuned.

Similar adaptive procedures as the ones described hereabove have been developped for this kind of control law.

\section{Dynamic controller in rational form}

The controller structure is a linear dynamical system described par its tranfer function:

$$
K(s)=\frac{N(s)}{D(s)}
$$

where $N$ and $D$ are polynomial w.r.t. the Laplace variable $s$. In practice this controller is aftenly implemented as an ARMA filter (and sometimes in a state space form). The parameters of the control law are the coefficients of the polynomials $N$ and $D$.

The paper will emphasis on the fact that such a control structure is very difficult to adapt online while acheiving closed loop stability. Indeed, the controller becomes instable when the measurement $y$ is stabilized because there is not enougth information. Bursts of instability appear.

\section{Dynamic controller in lattice form}

The Lattice form is another way to implement linear dynamic filters. Regalia [] has proposed several efficient tools to adapt such filters while preserving stability of the filter. Here we use these tools to adapt a controller in a feddback loop.

We show that some attention must be paid to avoid some unexpected behaviors. This results in a two stage adaption procedure. One stage adapts the static gain of the controller. The other stage adapts the dynamical part of the controller.

\section{Simulation results}

Few results of the simulation of previous methods are presented in this section. The next figures show the time history of the control $u(t)$ and the output $y(t)$.

In figure 6, method A corresponds to a fixed gain and delay. The output $y(t)$ is stabilized, but correct computation of the parameters is needed, and so an exhaustive knowledge of the system. In figure 7, method $\mathrm{B}$ corresponds to a fixed gain, but a variable delay. The solution is not converged and is unstable. The fixed gain needs also to be tuned properly, as explained in the previous method. In figure 9, the lattice shape, and also the gain, are updated in adaptive control. This method provides the more stable configuration and the output is fully stabilized. Little system knowledge is required (only the time scale).

\section{Concluding remarks}

Closed-loop feedback control was considered for the stabilization of a morphing wing flow by trailing edge vibration. A direct adaptive control is applied to a low-order dynamical oscillator. A Van der Pol nonlinear oscillator coupled with a chaotic May-Feigenbaum attractor is used as a representative model of the flow. Simulations tests show significant results obtained using a lattice filter and an adaptive gain. A complete stabilization of the output was performed with little information needed of the system.

\section{Acknowledgments}

This work is supported by the European project H2020 Smart Morphing and Sensing SMS (http://www.smartwing.org/SMS/). 

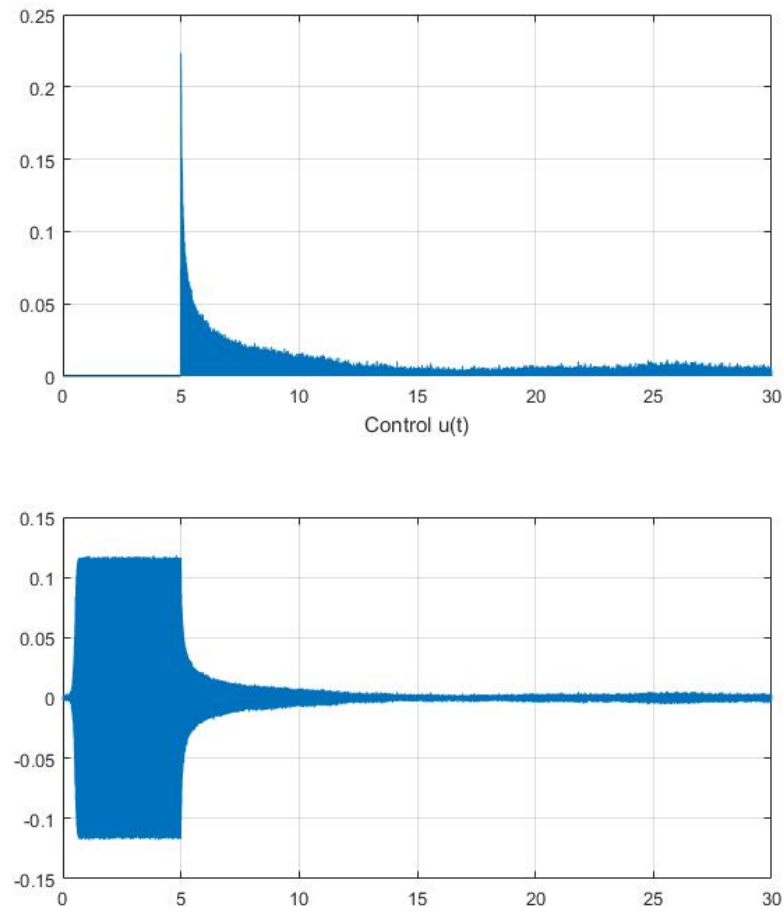

Figure 6: Method A: fixed gain and delay.
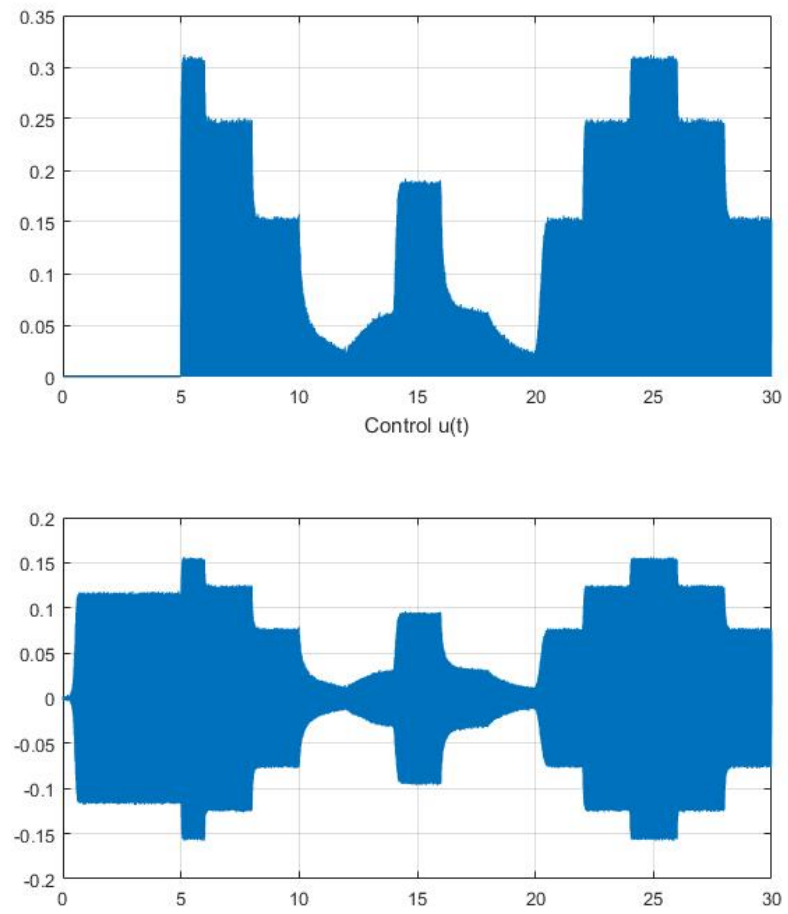

Figure 7: Method B: fixed gain, variable delay. 

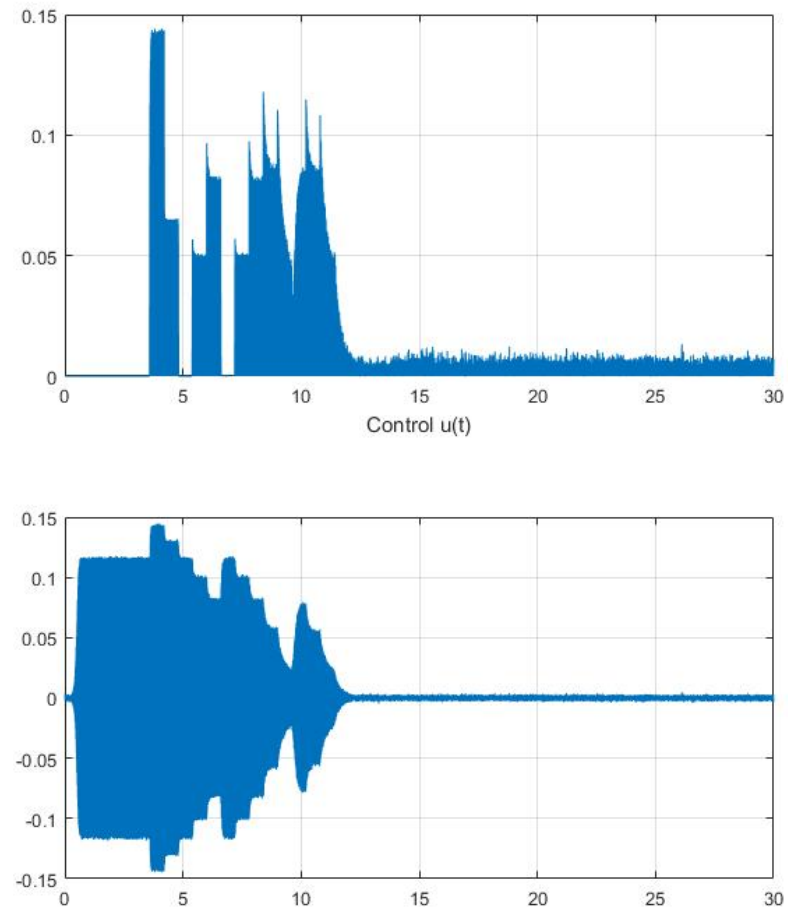

Figure 8: Method C: Optimization using gain exploration, fixed delay.
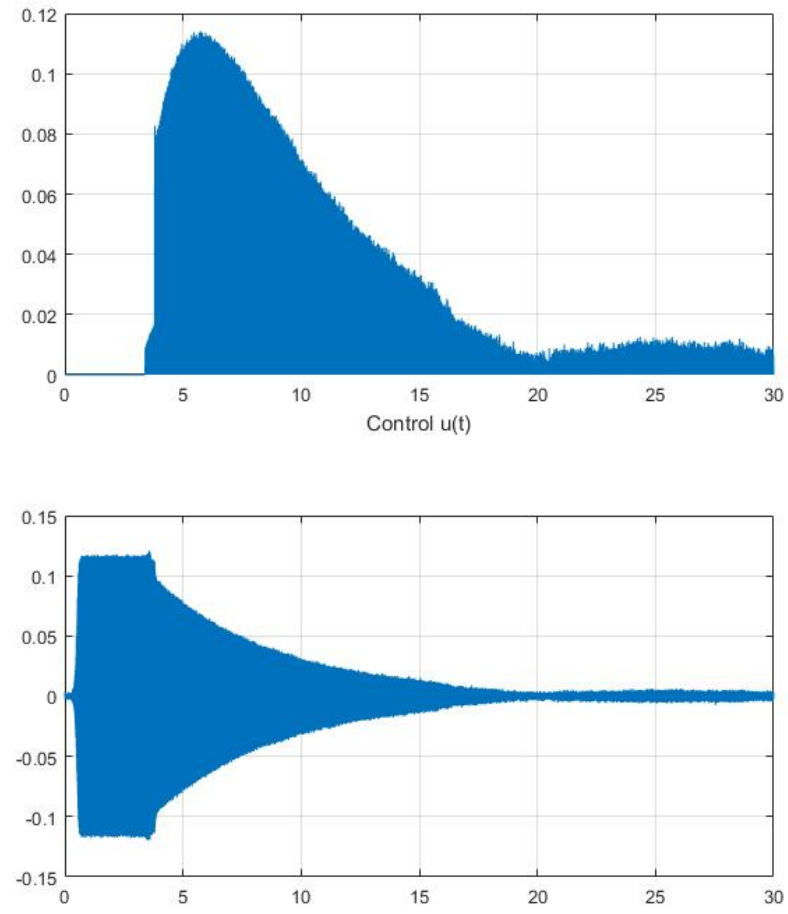

Figure 9: Method D: Lattice and adaptive gain. 


\section{References}

1 Gad-el hak, M. Turbulence Control and Applications. 2010.

2 Lin, J. C. Progress in Aerospace Sciences 2002, 38, 389-420.

3 Marquillie, M.; Ehrenstein, U.; Laval, J.-P. Journal of Fluid Mechanics 2011, 681, 205-240.

4 Scheller, J.; Jodin, G.; Rizzo, K. J.; Duhayon, E.; Rouchon, J. F.; Triantafyllou, M.; Braza, M. Solid State Phenomena 2016, 251, 106-112.

5 Jodin, G.; Motta, V.; Scheller, J.; Duhayon, E.; Döll, C.; Rouchon, J. F.; Braza, M. Journal of Fluids and Structures 2017, 74, 263-290.

6 Garwon, M.; Darmadi, L.; Urzynicok, F.; Bärwolff, G.; King, R. Adaptive control of separated flows. 2003.

7 Tian, Y.; Cattafesta, L. N.; Mittal, R. Adaptive Control of Separated Flow. 2006.

8 King, R.; Becker, R.; Feuerbach, G.; Henning, L.; Petz, R.; Nitsehe, W.; Lemke, O.; Neise, W. Adaptive flow control using slope seeking. 2006.

${ }^{9}$ Henning, L.; Becker, R.; Feuerbach, G.; Muminovic, R.; King, R.; Brunn, A.; Nitsche, W. Proceedings of the Institution of Mechanical Engineers. Part I: Journal of Systems and Control Engineering 2008, 222, 309-322.

10 Fliess, M.; Sira-Ramírez, H. Advances in Industrial Control 2008, 363-391.

11 Widrow, B.; Lehr, M.; Beaufays, F.; Wan, E.; Bilello, M. Adaptive signal processing. 1993.

12 Kreisselmeier, G. Automatica 1985, 21, 425-431.

13 Motta, V.; Mouyon, P.; Döll, C. Discrete Time Open-Loop and Closed-Loop Flow Control Based on Van der Pol Modeling. 2016; http://arc.aiaa.org/doi/10.2514/6.2016-3256.

14 Provensale, M.; Mathis, C.; Boyer, L. Journal of Fluid Mechanics 1987, 182, 1-22.

15 Khalid, M. S. U.; Akhtar, I. 2014, 1-23. 